\title{
Effect of Shaft Diameter of Pile on Lateral Winkler Spring Stiffness
}

\author{
A.Bahrami ${ }^{1}$ and H.Nikraz ${ }^{2}$ \\ Civil Engineering Department, Curtin University of Technology, Bentley Campus, Western Australia
}

\begin{abstract}
:
Soil was modelled with linearly elastic three dimensional finite elements. Lateral spring stiffness was calculated from FE results. Spring stiffness is shown varying linearly with shaft diameter. It is also found that spring stiffness is inversely proportional to powers (less than unity) of pile flexural rigidity. Scaling factors for shaft diameter and pile flexural rigidity are introduced. Basic stiffness of lateral spring is studied considering both full vertical slippage and zero slippage between the soil and the pile. Relevant relationships for stiffness are proposed. Some applications are suggested. Some applications are suggested.
\end{abstract}

KEY WORDS: Pile lateral stiffness, diameter effect on pile stiffness, soil pile interaction, Winkler spring stiffness, lateral spring stiffness of piles

\section{Introduction}

Piled foundations have been the subject of study for the past few decades. The estimation of deflection is of vital importance where deformation sensitive structures or machinery are to be supported. Structures such as nuclear plants are highly sensitive to deformation. Earthquake induced deformations in particular should be kept within a small range for these type of structures. Vibrating machinery is also sensitive to foundation movements. If a foundation's natural frequency is close to the operating frequency of such machinery, considerable amplification of deformation may take place in the foundation. In both examples above, the initial stiffness of pile constitute the parameters of the problem.

Correct estimation of pile head stiffness is a prerequisite for performing any lateral static or dynamic analysis of structures supported by piled foundations. This estimation is also essential in evaluating the free vibration frequencies of the structure with regard to resonance conditions produced by the earthquake or machinery induced vibrations.

The theory of Beam on Winkler's foundation was one of the earliest models used to study the problem of soil-pile interaction. Despite its shortcomings with regard to representing soil as a three dimensional continuum, this method is known for its simplicity and its adoptability under diverse site and soil conditions. For deflection controlled problems, soil behaviour may be considered elastic within a reasonable range of deformation and the Beam on elastic Winkler spring model can be used for the estimation of deformations and essential dynamic characteristics such as free vibration frequencies. Although the stiffness of the lateral Winkler spring has been the subject of research for decades and a number of explanations have been proposed by different investigators as to the true nature of the lateral spring, this is still a matter under discussion.

Debates on the effect of pile diameter have been ongoing since research began and they continue to date. Terzaghi (1955) proposed that the stiffness of beam on elastic foundation should be directly proportional to beam width, and consequently the spring constant becomes independent to width. Vesic's (1961) expression of the modulus of subgrade reaction agreed with this discussion. Gazetas (1991) proposed expressions based on finite elements studies, where pile head stiffness is directly proportional to pile diameter. Novak and El Sharnoubi (1983) also proposed expressions based on continuum mechanics studies which showed direct proportionality of pile head stiffness to diameter. It has become a common assumption that lateral spring stiffness is directly proportional to shaft diameter, i.e., as the diameter is doubled, so is the stiffness; and as the diameter is made smaller the stiffness vanishes accordingly. This conclusion is incorrect, as is in the case of a very small (mathematically zero) diameter pile, the stiffness should approach the limiting value of an elastic half-space loaded with a horizontal point force on its free surface (e.g. Johnson 1985). Carter (1984), Pender (1993) and Ling (1988), on the other hand, reported direct proportionality between spring stiffness and shaft diameter based on full-scale pile test results. Ashford and Juirnarongrit (2003) tested this hypothesis by back-analysing free vibration measurements on full scale test piles with both diameter dependent and diameter independent relationships to spring stiffness. They concluded that diameter independent springs produce results which are closer in correlation to their measurements. Pender and Carter et al. (2007) have argued that by increasing shaft diameter, the extension to which the soil is contributing to pile stiffness would be increased, and therefore the stiffness of the springs would increase as pile diameter increases.

In this technical note, variations of both pile lateral stiffness and Winkler spring initial stiffness with shaft diameter of pile were investigated. Large numbers of finite element analyses were performed to create a database which covered a wide range of possible variations in material and configuration of the soil-pile system. Variations in pile stiffness and spring stiffness were investigated by using data analysis techniques.

\section{Finite Element Models}

Using finite element method, the soil is modelled with elastic-homogenous, three dimensional cubic elements. The thickness of the soil layer is $10 \mathrm{~m}$ and extends $29.5 \mathrm{~m}$ from each side of the pile. Examination shows that changing the far end restraint conditions of the modelled soil does not significantly affect the calculated head stiffness of the pile. ${ }^{1}$ Amir Bahrami MIEAust, Ph.D. Civil Engineering
amir.bahrami@postgrad.curtin.edu.au, Mobile $:(+61) 425638272$

${ }^{2}$ Professor at Civil Engineering and Head of Civil Engineering Department, Curtin University

E-mail: H.Nikraz@curtin.edu.au 
Therefore the extension of the modelled soil media is assumed to satisfactorily represent soil stiffness properties. The limited length of the pile and the fixed boundary on the bottom may give rise some concerns regarding the analogy with the beam on Winkler's foundation which is based on flexible piles with virtually infinite length. Therefore it should be noted that the main analogy made in this paper is based on a solution for finite length beam. A contingent condition for this solution to correspond to that regarding an infinite beam is investigated within the text. It is observed that with the exception of very soft soils and a very low slenderness ratio of pile, these two solutions result in the same figures. A rule of thumb exists for the fixity length of the pile to be 5 times its own diameter. Considering that the modelled depth of pile is 10 meters, it is anticipated that piles with a diameter of $1 \mathrm{~m}$ or less should represent a flexible pile with reasonable accuracy. It is also assumed that any inaccuracies would not be reflected on the shaft diameter-related part of Winkler spring stiffness due to the specific formulation used in the text.

Only one quarter of the space is modelled, due to existing symmetry with respect to the "y-z" plane (i.e., the plane of lateral deflection of pile). It is evident that each quarter-space, which is bounded by "xz" and "yz" planes, includes exactly the same number of brick elements and the same shape of quarter-cylindrical cavity. It can therefore be concluded that the stiffness of a quarter space will be exactly one fourth of that of the full space provided that the connectivity between the pile and the soil is similar in every direction. It should be noted that this argument is only valid from the stiffness point of view when attributed to pile elements; it does not apply to the stresses and strains in soil elements. To successfully replace the full-space model with a quarter-space model, some artificial restraints on artificial boundaries must be formed to correctly simulate the effect of the eliminated parts from the model. Consider three deformation components in soil in cylindrical coordinates " $u_{r}, u_{\theta} u_{z}$ " as functions of $(r, \theta, z)$. It can be concluded from the symmetry with the " $\mathrm{y}-\mathrm{z}$ " plane that " $u_{\theta}(r, \theta, z)=-u_{\theta}(r, \pi-\theta, z), u_{r}(r, \theta, z)=u_{r}(r, \pi-\theta, z)$ ". Noting that deformation components should be periodic functions of " $\theta$ ' with period " $2 \pi$ ", it can be concluded that a Fourier series expansion of " $u_{\theta}$ " with respect to " $\theta$ ' may contain only cosine terms and for " $u_{r}$ " only sine terms. Assuming connectivity between horizontal deformations of pile and those in the soil, it can be written that " $u_{r}(D / 2,0, z)=u_{x}=0, u_{\theta}(D / 2,0, z)=u_{y}=Y(z)$ ", where "Y(z)" holds for the lateral deflection of the pile axis. In addition, it is known that deformation components must 'disappear' at a far distance from the pile in a semi-infinite space. Therefore the radial deformation component has two zeroes alongside "x-Axis", one on the pile surface and another one at infinity. The conclusion is that this deformation component should be zero everywhere on the "xz-plane", since it cannot have a non- zero value after its initial zero value on the pile surface and it again goes to zero at far distances (in the absence of applied loads in between). A similar argument is valid for the tangential component of deformation on the "yz-plane". Therefore artificial boundary surfaces, which are formed by "xz" and "yz" planes, should be restrained for deformation in "x" direction. A full model FE analysis is made and the force-deflection ratio at the pile's top proved to be one fourth of the quarter-space model. The bottom surface of the modelled soil is restrained against movement thereby modelling rigid bedrock. The presence of the pile shaft is modelled as a quarter cylinder cavity in the FE model for soil elements. A $3 \mathrm{~m} \times 3 \mathrm{~m}$ transition region is introduced to transfer from very fine mesh on the pile shaft surface to a uniform mesh of $0.5 \mathrm{~m} \times 0.5 \mathrm{~m} \times 0.5 \mathrm{~m}$ which extends to the rest of the model. A total number of 71,060 three-dimensional elements are used in modelling the soil. Pile is considered as a solid (non-tubular) circular cross-section with a typical Young's modulus of elasticity of 25000 $\mathrm{MPa}$. Classic Euler-Bernoulli elements are used to model the pile. Since only a quarter of the soil is modelled, crosssectional properties of the pile are also multiplied with 0.25 . Full connectivity between the soil and the pile at the pile's shaft surface is provided via a number of rigid, one-dimensional elements which connect the pile's centreline nodal points to relevant points of the soil elements in all horizontal directions. Previous but unpublished work of the authors on an elastic continuum mechanics model of pile under lateral load on half space media shows that only the horizontal components of the deformations in the soil and the pile on their contact surface can be equated. Their vertical components however, cannot be made equal with the exception of a rare case where Poisson's ratios of soil and pile material are equivalent. Therefore, even in a perfectly elastic model, vertical slippage between the soil and pile surface, to some degree, would be inevitable. In reality, this slippage might be affected by factors like pile surface conditions, soil type and magnitude of strain in soil. Modern finite element softwares are equipped with features like gap elements and links which allow such factors to be included in the model. The numerical values for the essential characteristics of such factors are only justifiable for specific types of soil and the condition of the pile surface. It would be impractical as well as unrealistic to perform a large number of FE analyses on a wide range of possible soil mechanical characteristics with the abovementioned factors included. For the aim of this study, a homogeneous elastic assumption for material with simple connectivity between pile and soil which enables a large number of FE analyses is more suitable although, qualitative and quantitative evaluation of the upper and lower limits of soil-pile connectivity conditions is necessary to address the effect of relative slippage. Full slippage between pile and soil represents the lower limit of the problem, as it leads to an underestimation of pile lateral stiffness. A finite element model with such connectivity shows zero vertical deformation throughout all elemental nodal points. A fully connected model, on the other hand, gives an overestimate of pile lateral stiffness. Knowing that conditions in the field will be somewhere in between these two extreme cases, both types of conditions are investigated in the present study. The results are in the form of upper and lower limits springs stiffness via which sensitivity of the overall structural behaviour can be investigated for any particular case.

The pile tip is restrained against movement and rotation to resemble an end-bearing pile with full penetration into bedrock. A horizontal force in "y" direction is applied to the pile's top and static analyses are performed. Figure 1 shows the configuration of the finite element model used in this study. 


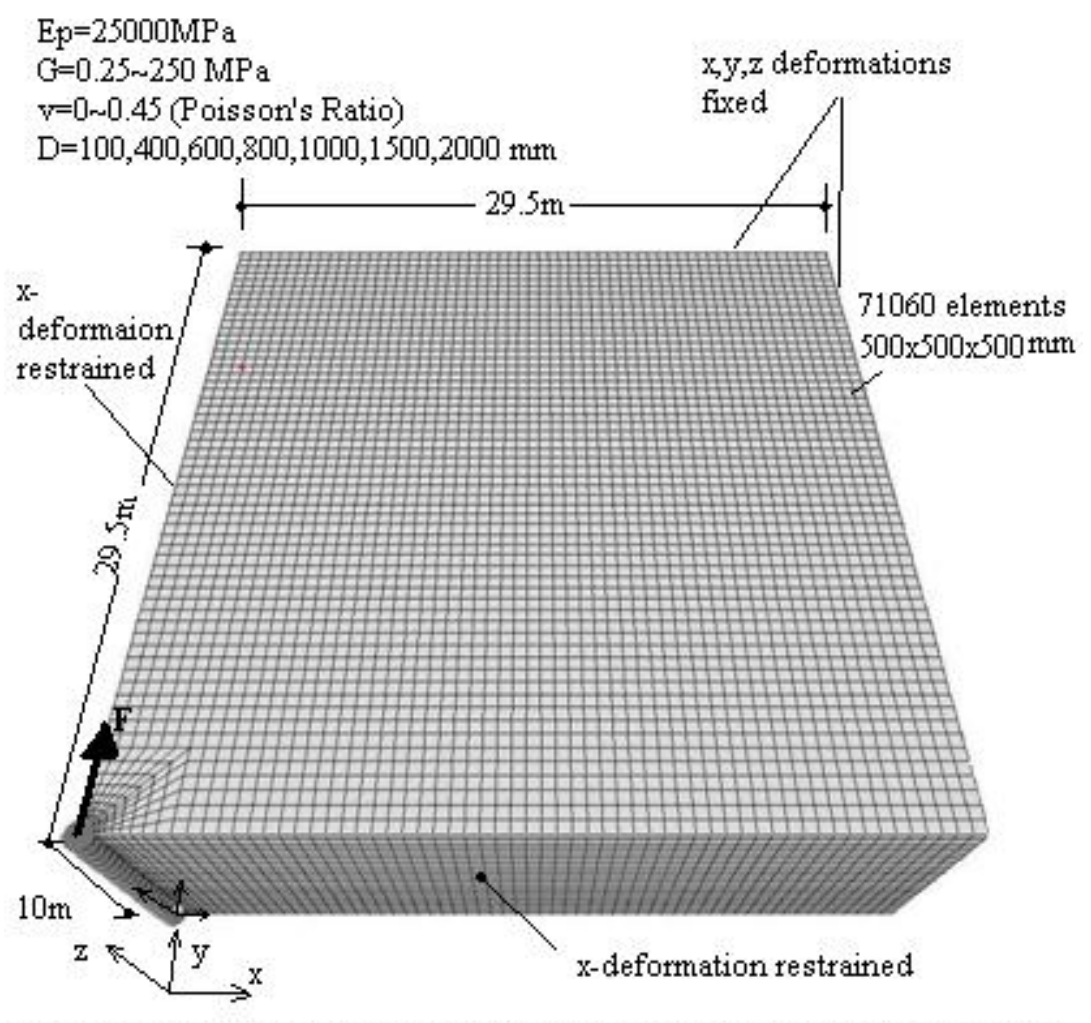

Figure 1-3D finite element model of $1 / 4$ soil half space and pile in the comer

Static analyses with different configurations and material characteristics are performed to create databases. The first set of FE models is created with shaft diameters of 100, 400, 600, 800, 1000, 1500 and 2000 millimetres. Each model, which has a specific shaft diameter, has been analysed for modulus ratios of 100, 250, 500, 1000, 2500, 5000, 10000, 20000,50000 and 100000 . Pile flexural rigidity " $E_{p} I_{p}$ " is kept constant and equal to that of a circular cross- section with $D_{\text {Ref }}=1 m$ for the first set of 60 FE models.

The second set of FE models are created by changing the piles' second moment of inertia in the first set of models to " $I_{p}=\pi D^{4} / 64 "$ " (D = shaft diameter $)$.

In all FE models, the head of the pile is free to rotate about the " $x$ " axis and to translate in both " $y$ " and " $z$ " directions. Many full-scale pile tests, from which lateral spring stiffness is derived, are performed in similar free head conditions. Therefore the choice of these types of end conditions makes the results of this study more practicable for use in the field.

Pile free head stiffness is calculated from the FE results by dividing the applied horizontal force by the horizontal displacement at the pile's top. Since the one quarter model possesses only one quarter of the stiffness of the real soil pile system, this ratio is then multiplied by a factor of 4 to obtain the true stiffness of the pile. Figure 2(a) shows the variation of pile head stiffness, with the shaft diameter of the pile with pile flexural rigidity kept constant. According to this Figure, the free head stiffness of the pile varies linearly with the shaft diameter. This linear aspect is almost ideal for softer soils, while some deviations are observed for stiffer soils. The intersections of the lines with the vertical axis are the results of FE models made without a cylindrical cavity (i.e., with zero shaft diameter). The fact that these lines are not passing through the point of origin proves that the lateral spring stiffness is not independent of the shaft diameter.

Figure 2(b) depicts the results of the second set of analyses with exactly the same FE configuration but with the second moment of inertia of pile varied correspondingly to shaft diameter. The resulting curves for pile stiffness reflect the combined effect of shaft diameter and pile flexural rigidity on the free head stiffness of the pile. Deviation from the straight line is clearly observed for the curves in Figure 2(b). The intersections of lines with a vertical axis are derived from FE analyses without a central cavity (i.e., with zero shaft diameter) and no flexural elements representing pile. These points are representative of the resistance shown by an elastic half space against a tangential concentrated force applied to its surface. This class of problems is theoretically investigated by Boussinesq and Cerruti (e.g. see Love 1944) and its application is found in contact mechanics (e.g. Johnson 1985). A Boussinesq type solution is not capable of calculating deformations at the exact point of the application of a concentrated load due to the presence of singularites in mathematical model. Therefore, a theoretical representation of so-called lateral stiffness for an elastic half space under a tangential load is not achievable. The finite element method however, due to the implementation of non-singular shape functions and other aspects, provides finite displacement at the application of a concentrated load from which pseudo-stiffness for the media can be defined. Another important consideration is that these points follow the course of their corresponding curves perfectly. It proves that they are neither artefacts of the finite element model nor a result of numerical approximations. Therefore they can be relied on as limiting values of the stiffness of the pile when the shaft diameter is vanishing. It should be noted that the first and second sets of FE models share the same depth and similar boundary conditions at the bottom. It is concluded from the above discussion that the nonlinear shape of the curves in Figure 2(b) cannot be a result of boundary conditions and finite pile length, as they are equivalently present 
for data in Figure 2(a). The authors believe that this nonlinearity is a result of the combined action of shaft diameter and pile flexural rigidity. The fact that the latter varies with the forth power of the shaft diameter justifies the nonlinear nature of the curves in Figure 2(b).
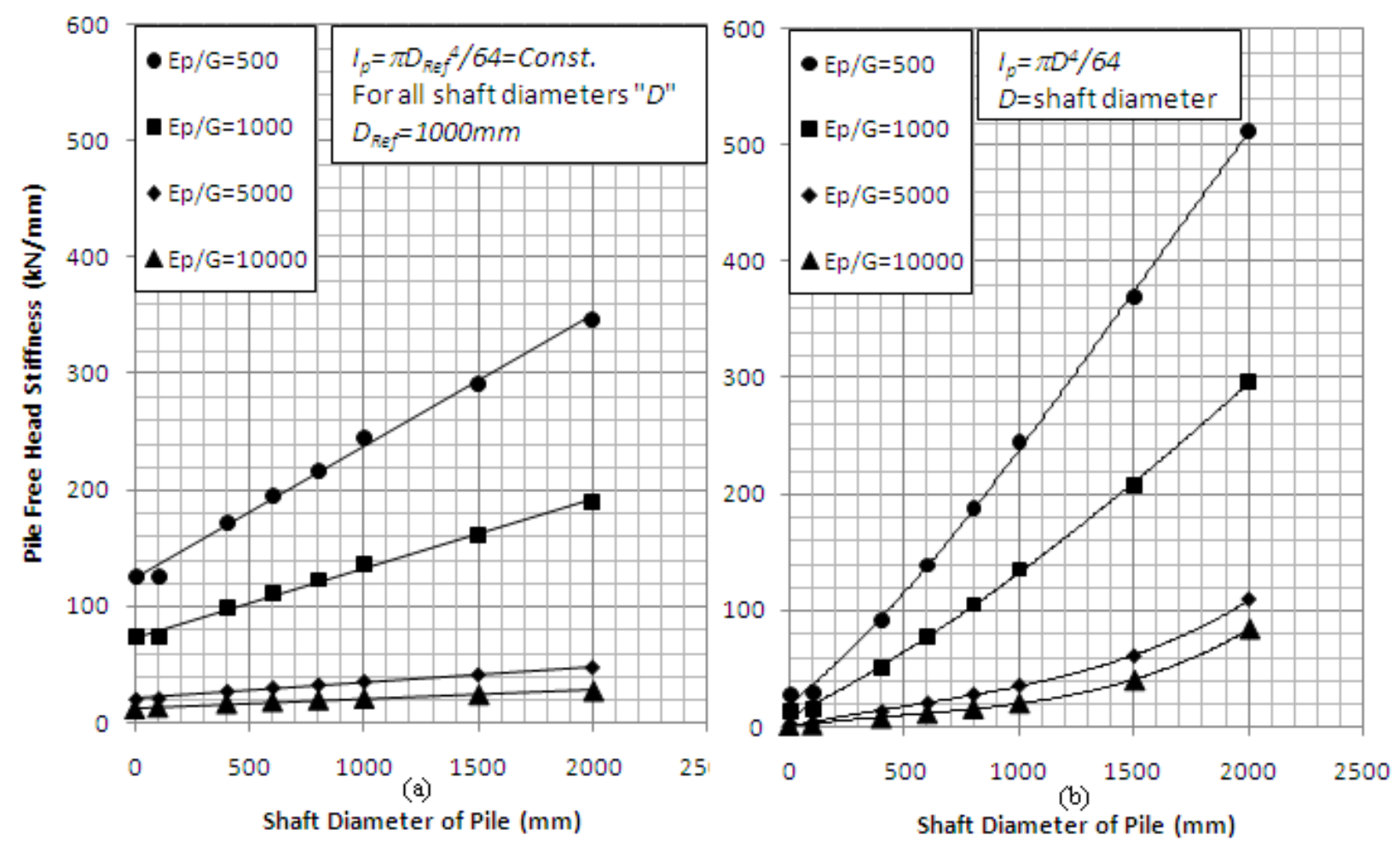

Figure 2- Variations of pile free head stiffiness with shaft diameter:

a) Pile second moment of inertia is (artificially) kept constant for all different shaft diameters in FE models.

b) Pile second moment of inertia is equal to that of a soilid circular (non-tubular) section fit in the shaft cavity.

\section{Winkler Spring Stiffness}

An important implication of the information in Figure 2 is that the lateral stiffness of the pile is not directly proportional to the pile diameter. This aspect is more clearly observed for soft soils. Therefore it is anticipated that Winkler spring constants, in these kinds of soils, show dependency on diameter. It is also expected that unlike pile head stiffness, which reflects the global interaction of half space soil with pile, the Winkler springs stiffness relate to local configuration parameters like shaft diameter and pile flexural rigidity. This feature makes the study of spring stiffness worthwhile from a practical point of view in that the results of such a study may be more readily adapted for use in variable soil parameter sites, layered soils and other appropriate arenas.

For the purpose of determining spring stiffness, classic theory of beam on elastic foundation is employed in which soil is modelled as linearly elastic lateral springs. Pile is ordinary Euler-Bernoulli beam with a finite length, "H", resting on rigid bedrock. The pile is assumed to have enough penetration into the bedrock to be considered as fixed at its tip.

Pile lateral deflection is arbitrarily assumed to take place in a horizontal " $y$ " direction. The pile axis is assumed to be in a vertical " $z$ " direction, with the origin at the pile head and in a positive downwards direction. The effect of the axial load is ignored for simplicity. Governing differential equation is as follows:

$$
E_{p} I_{p} \frac{d^{4} Y(z)}{d z^{4}}+K_{s} Y(z)=0
$$

Where

$K_{s}=$ Lateral initial Winkler spring constant

$H=$ Soil layer thickness $=$ pile embedded length

$E_{p}=$ Young modulus of elasticity for pile material

$I_{p}=$ Pile cross-section second moment of area

For a pile with a finite length " $H$ ", clamped at its tip with a horizontal force " $F_{0}$ ", acting at its top at the ground level, the solution for (2) can be written as:

$$
Y(z)=\frac{F_{0}}{2 E l \beta^{3}}\{A \sin [\beta(H-z)] \cosh [\beta(H-z)]+B \sin (\beta z) \sinh [\beta(H-z)]+C \cos [\beta(H-z)] \sinh [\beta(H-z)]\}
$$

Where " $A, B, C$ " are constants to be determined based on end conditions (note that Eqn.(2) already satisfies the end condition on the pile tip, i.e., zero movement on the bedrock " $y(H)=0$ ”) and:

$\beta=\sqrt[4]{\frac{k_{s}}{4 E_{p} I_{p}}}$

Since most pile tests are performed with free head (i.e., zero bending moment on the pile head), coefficients; A, B and $\mathrm{C}$ might be evaluated by considering free head conditions: " $M(0)=E_{p} I_{p} y^{(2)}(0)$ " and " $V(0)=F_{0}=E_{p} I_{p} y^{(3)}(0)$ " and fixed 
tip condition ' $y$ ' $(H)=0$ ”. In literature however, these constants are also determined for two other sets of conditions, i.e. head translation with zero rotation as well as head rotation with zero translation. These cases are not considered in the present article, for the sake of brevity.

Having determined $\mathrm{A}, \mathrm{B}$ and $\mathrm{C}$ for free head conditions, the ratio of a horizontal force " $F_{0}$ " to the resulting top translation " $Y(0)$ ", can be considered as free (unrestrained) head lateral stiffness " $K_{h}$ ":

$K_{h}=\frac{2}{3}(\beta H)^{3} \frac{\cosh (2 \beta H)+\cos (2 \beta H)+2}{\sinh (2 \beta H)-\sin (2 \beta H)}\left(\frac{3 E_{P} I_{P}}{H^{3}}\right)$

Expression (4) describes the free head lateral stiffness of a single pile as a fraction of free head cantilever stiffness with the length " $H$ " and the flexural rigidity " $E_{p} I_{p}$ ". In cases of very soft soil it is expected that (4) approaches the stiffness of a free head cantilever. This can be mathematically investigated by taking the limit of (4) for " $\beta H \rightarrow O$ " with three times the application of L'Hospital's rule (see e.g. Pipes and Harvill 1970). This conclusion can be used to interpret the nonlinear curves in Figure 2(b) for softer soils. For this kind of soil the pile head stiffness becomes closer to that of a cantilever which is related to higher powers of diameter.

For very slender piles, i.e., long piles with a relatively small diameter, another solution to (1) can be established as:

$$
Y(z)=\frac{F_{0}}{2 E I \beta^{3}} e^{-\beta z} \cos (\beta z)
$$

Pile stiffness can be derived from (5) for flexible piles as:

$K_{h}=2 E_{P} I_{P} \beta^{3}$

It can be proved that (4) mathematically approaches (6) if its limit is taken as " $H \rightarrow \infty$ ". Practically, for " $\beta H>5$ ", equations (4) and (6) effectively result in the same figures.

Factor " $\beta H$ " can be calculated by substituting values for " $K_{h}$ " from FE analyses in equation (4). This equation is chosen over equation (6) in order to take into account the effect of limited pile length. Spring stiffness can then be calculated by substituting " $\beta H$ " from the previous step in (3). For flexible piles, a more straightforward relationship can be obtained by eliminating " $\beta$ ' between (6) and (3) which gives more insight into the methodology of the present study:

$k_{s}=\sqrt[3]{\frac{4 K_{h}^{4}}{E_{p} I_{p}}}$

It is evident from (7), that if pile head stiffness is proportional to shaft diameter, spring stiffness becomes independent of shaft diameter. However, such a conclusion is only valid for flexible piles where $\beta H>5$ as occurs in either stiff soils or long piles.

It is assumed that lateral Winkler spring stiffness can be written as basic stiffness modified with scalings or allowances for the shaft diameter and flexural rigidity of the pile as scaling factors. Basic stiffness is assumed to be a function of soil mechanical characteristics (i.e., shear modulus " $G$ " and Poisson's ratio “ $v$ ") and the modulus ratio " $E_{p} / G$ ". It is also assumed that basic stiffness is not affected by the shaft diameter and flexural rigidity of the pile and this can be written as:

$k_{s}=\left(k_{0}\right) S_{D} S_{I}$

Where:

$$
\begin{aligned}
& k_{0}=\text { Basic spring stiffness. } \\
& S_{D}=\text { Diameter scaling factor. } \\
& S_{I}=\text { Flexural rigidity scaling factor. }
\end{aligned}
$$

The focus of the present study is to determine the scaling factors " $S_{I}$ " and " $S_{D}$ " from FE analyses results. To eliminate the effect of basic spring stiffness from the analysis data, it is enough to divide pile stiffness values by the relevant values of a reference pile. In this study, a pile with the shaft diameter " $D_{\text {Ref }}=1 \mathrm{~m}$ " is chosen as the reference pile.

To determine the diameter scaling factor " $S_{D}$ ", the first set of FE results (those with constant pile flexural rigidity) is divided by the spring stiffness for the reference pile. This will eliminate " $\mathrm{k}_{0}$ " and " $\mathrm{S}_{\mathrm{I}}$ " and the remaining will be " $\mathrm{S}_{\mathrm{D}}$ ". Figure 3 shows variations of the diameter scaling factor with the diameter ratio. It can be seen that the values of " $S_{D}$ " show insignificant variability with the modulus ratio " $E_{p} / G$ ". Therefore curve fitting could be applied using average values. A simple expression can be proposed for " $S_{D}$ ", with acceptable accuracy, for " $D / D_{\text {Ref }}>0.4$ " as:

$$
S_{D}=0.5\left(1+D / D_{\operatorname{Re} f}\right)
$$

To obtain the flexural rigidity scaling factor, a similar procedure is followed with the second set of FE analyses results, dividing the calculated spring stiffness by those for the reference diameter (i.e., " $D_{R e f}=1 \mathrm{~m}$ ") will result in the product " $S_{D} S_{I}$ ". Dividing this product further by the " $S_{D}$ " factors, which were determined in the previous step, results in the flexural rigidity scaling factor " $S_{I}$ ". Data analysis indicates that the flexural rigidity scaling factor can be expressed as:

$S_{I}=\left[\frac{\left(E_{p} I_{p}\right)_{\mathrm{Re} f}}{E_{p} I_{p}}\right]^{\gamma}$

Where " $\gamma$ " is a power that varies from 0.15 for very stiff soils to 0.036 for very soft soils and can be expressed as: 
It is evident that unless pile diameter differs greatly to the reference diameter, the scaling factor " $S_{I}$ " is not significantly different from the unity. Therefore this factor will be of secondary importance compared to the diameter scaling factor.

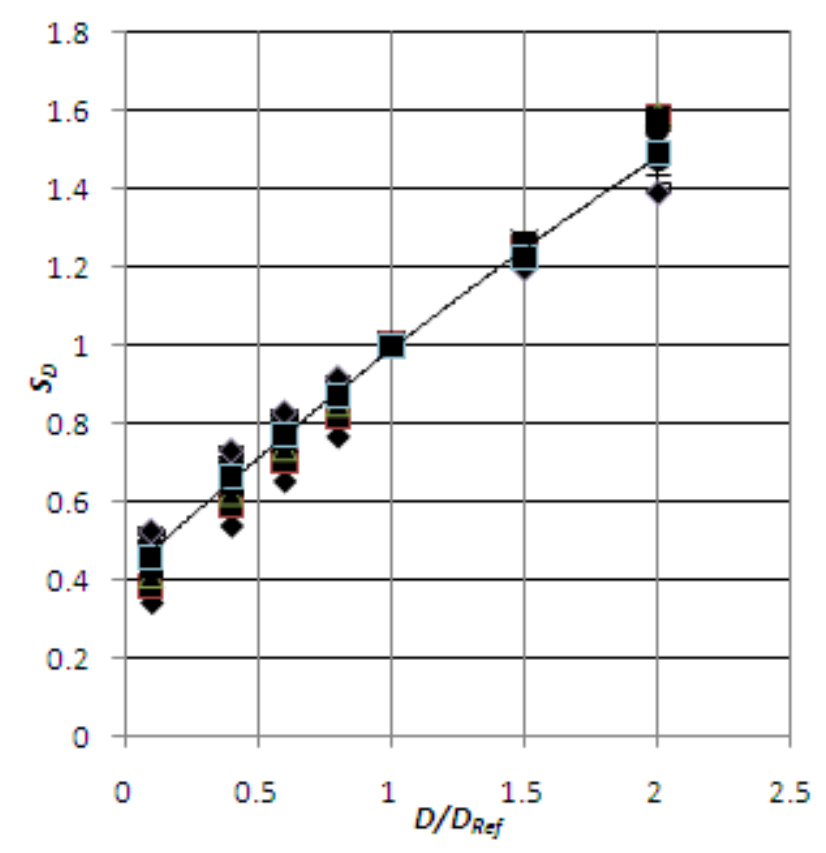

Figure 3- Variation of Diameter correction factor with diameter ratio

It is assumed that errors due to simplifications in soil pile connectivity and bottom boundary conditions are reflected in the basic stiffness factor " $k_{0}$ ". In addition, it is expected that the methodology which is used in the determination of " $S_{D}$ " and " $S_{I}$ " factors eliminates such errors from these factors. From this point of view, the diameter and flexural rigidity scaling factors are assumed to be unaffected by the boundary and connectivity conditions and their application to practical situations may be justified.

Data obtained from FE analyses has also been used to determine the basic stiffness " $k_{0}$ ", for both upper and lower limit cases described before. The procedure is briefly reported here.

The FE models with reference diameter are analysed for different Poisson's ratios “ $0,0.2,0.4,0.45,0.48$ ”. Spring stiffness is derived using equations (3) and (4). The resulting values are divided with relevant stiffness values for zero Poisson's ratio. By curve- fitting the results, an expression for the variations of spring stiffness with Poisson's ratio is derived. Stiffness values for zero Poisson's ratio are then correlated with the modulus ratio "G/E ". The following shows the final result for the upper limit conditions (i.e. full connectivity between soil and pile at the shaft surface):

$\left(k_{0}\right)_{u}=G\left[2.86+20.15\left(\frac{G}{E_{p}}\right)^{0.41}\right]\left[1+\left(\frac{G}{50 E_{p}}\right)^{0.0678} \frac{v}{1-v}\right]$

The above procedure is repeated with vertical deformation component released between nodal points of pile and those for the soil elements on shaft surface. This will resemble full slippage on the pile shaft while lateral connectivity is maintained. Far end boundaries are also released to contradict the upper estimation which has been included in the previous sets of analyses by fixing these boundaries. The following equation is proposed for the lower limit basic spring stiffness as a result of curve fitting:

$\left(k_{0}\right)_{1}=G\left[2.65+9.10\left(\frac{G}{E_{p}}\right)^{0.40}\right]\left[1+\left(\frac{G}{50 E_{p}}\right)^{0.0678} \frac{v}{1-v}\right]$

An average value will clearly eliminate any effect of far end restraint conditions which might be inevitably included in equations (12) and (13). It also may provide a more realistic view about the effect of slippage on spring stiffness:

$\left(k_{0}\right)_{\text {ave }}=G\left[2.75+14.62\left(\frac{G}{E_{p}}\right)^{0.40}\right]\left[1+\left(\frac{G}{50 E_{p}}\right)^{0.0678} \frac{v}{1-v}\right]$

Figure 4depicts variations of basic spring stiffness (for zero Poisson's ratio) with modulus ratio. As it is argued before, even in for a completely elastic soil slippage between soil and pile occurs, therefore using upper limit value of Eq.(12) for practical cases is not recommended. Lower limit and average basic stiffness may be used with engineering judgment on the amount of the slippage which needs to be included in a particular problem. 


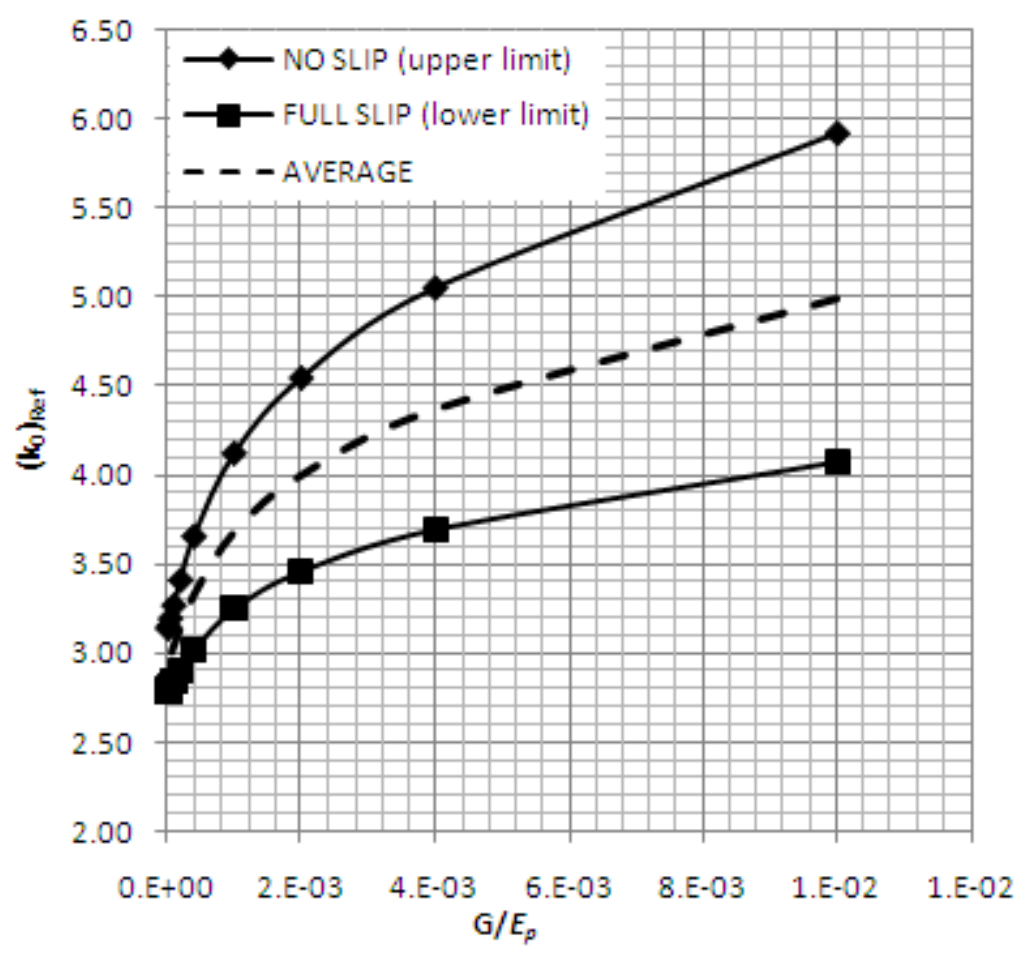

Figure 4 -Upper and lower limit basic stiffness of lateral Winkler springs

\section{Applications}

Aside from the insights that the results of this study provide, a number of applications can also be anticipated.

Assessment of seismic effects on buildings requires determination of fundamental periods of the structure (e.g. ASCE/SEI 7-05 2006). In piled supported structures, the fundamental periods are affected by the soil-pile interaction. Lateral and vertical spring stiffness can be used in a FE model to replace large media of three dimensional soil elements. Fundamental parameters to be input in such models are springs stiffness which can be evaluated by different means. Where the spring stiffness is determined from test pile data, which is not uncommon to be undersized, compared to final pile dimensions, the numerical values can be scaled and corrected by using the scale factors " $S_{D}$ " and " $S_{I}$ ". This will lead to a better and more accurate estimation of total stiffness of the model and consequently of the fundamental frequencies of the structure. Even when pile test data are unavailable, lateral spring stiffness can be estimated from equations (13) and (14) with special attention on magnitudes of the soil fundamental characteristics (i.e. shear modulus and Poisson's ratio) to be used for earthquake analysis. The upper limit and lower limits provided in equations (12) and (13) can also be considered as a basis to investigate the sensitivity of the structure with soil stiffness properties in response to earthquake vibrations.

If reasonable estimates of separation length and degree of slippage between the soil and the pile is available (e.g. based on previous experience or geotechnical investigation), the above formulas can be factored and combined accordingly to form a more realistically model the actual case. For example springs stiffness in the few first centimetres depth can be ignored or factored to resemble the separation between the pile and the soil. Lower limit formula (13) can be used where the pile surface is very soft or a full slippage is anticipated due to high strain field in the soil, e.g. at the first one thirds of the depth. For the rest of the depth the average formula (14) may be used. In the absence of full test data correlations, equations (12) to (14) might be used with care and good engineering judgement.

The similar concept is applicable for the design of sensitive structures like nuclear power plants and vibrating equipment foundations with regard to earthquake motions and machine vibrations. In these types of structures, deflections should be kept at very low levels to prevent damage to machinery and critical equipment. At such levels of deformation, soil will behave reasonably within elastic limits and the results of this present study will yield even more in practicality.

\section{References}

[1]. (ASCE/SEI 7-05 2006). Minimum Design Loads for Buildings and Other Structures. ASCE/SEI 7-052006. A. S. o. C. Engineers, American Society of Civil Engineers

[2]. Ashford, S. A. and T. Juirinarongrit (2003). "Evaluation of Pile Diameter Effect on Initial Modulus of Subgrade Reaction." JOURNAL OF GEOTECHNICAL AND GEOENVIRONMENTAL ENGINEERING ( ASCE 129(3): 234-242.

[3]. Carter, D. P. (1984). A non-linear soil model for predicting lateral pile response. C. E. D. Rep. No. 359, Univ. of Auckland, New Zealand. 
[4]. Gazetas, G. (1991). Foundation vibrations. Foundation Engineering Handbook, 2nd Ed. H.-Y. E. Fang and V. N. Reinhold. New York: 553-593.

[5]. Johnson, K. L. (1985). Contact Mechanics, Cambridge University Press.

[6]. Ling, L. F. (1988). Back analysis of lateral load tests on piles, Civil Engineering Dept., University of Auckland, Newzeland.

[7]. Love, A. E. H. (1944). A TREATISE ON THE MATHEMATICAL THEORY OF ELASTICITY. NEW YORK, DOVWER PUBLICATIONS.

[8]. Novak, M. and B. El Sharnouby (1983). "Stiffness Constants of Single Piles." Journal of Geotechnical Engineering 109(7): 961-974.

[9]. Pender, M. J. (1993). "A seismic pile foundation design analysis." Bull. NZ Nat. Soc. Earthquake Eng 26(1): 49-160.

[10]. Pender, M. J., D. P. Carter, et al. (2007). "Diameter Effects on Pile Head Lateral Stiffness and Site Investigation Requirements for Pile Foundation Design." Journal of Earthquake Engineering 11: $1-12$.

[11]. Pipes, L. A. and L. R. Harvill (1970). Applied Mathematics for Engineers and Physicists, McGraw-Hill Book Company.

[12]. Terzaghi, K. (1955). "Evaluation of coefficients of subgrade reaction." Geotechnique 5(4): 297-326.

[13]. Vesic, A. S. (1961). Beam on elastic subgrade and the Winkler hypothesis. Proc., 5th Int. Conf. Soil Mechanics and Foundation Engineering. Paris. 1: 845-850. 